Article

\title{
An Improved Stochastic Response Surface Method Based Probabilistic Load Flow for Studies on Correlated Wind Speeds in the AC/DC Grid
}

\author{
Ziwei Zhu ${ }^{1, *}$, Shifan $\mathrm{Lu}^{1}$ and Sui Peng ${ }^{2}$ \\ 1 Information Engineering College, Nanchang University, Nanchang 330031, Jiangxi, China; \\ lushifan@email.ncu.edu.cn \\ 2 Grid Planning \& Research Center, Guangdong Power Grid Corporation, CSG, Guangzhou 510080, \\ Guangdong, China; pengsui@cqu.edu.cn \\ * Correspondence: zhuziwei@tsinghua.org.cn
}

Received: 18 November 2018; Accepted: 12 December 2018; Published: 15 December 2018

\begin{abstract}
This paper proposed a probabilistic load flow technique of AC/VSC-MTDC (Alternate Current/Voltage Source Control-Multiple Terminal Direct Current) hybrid grids based on an improved stochastic response surface method. The applied traditional stochastic response surface method is inherent with the capability to tackle correlated normal variables; however, the accuracy is poor in the case of correlated diverse distributions. To address this issue, NATAF transformation was adopted to transform the correlated wind speeds and loads following arbitrary distributions into the variables that are subject to standard normal distributions. The collection points could be selected to establish the polynomial relationship among the independent standard normal variables and the output responses. Then, the probability distributions and statistics of the responses could be accurately and efficiently estimated. The modified IEEE 14-bus system, involving an additional VSC-MTDC system, wind speeds following various distributions, and diverse consumer behaviors, was used to demonstrate the validity and capability of the proposed method.
\end{abstract}

Keywords: probabilistic load flow; AC/VSC-MTDC hybrid grids; stochastic response surface method; correlation

\section{Introduction}

In modern power systems, the use of wind power is drawing great attention. By the end of 2020, wind power is predicted to provide $12 \%$ of the world's electricity [1]. Increasingly, many countries are choosing to build large-scale wind farms (WFs) in places with superior wind conditions [2]. Voltage Source Converter based High Voltage Direct Current (VSC-HVDC) is considered to be an effective solution for large-scale WFs because it has the ability to independently control the active and reactive power and to support voltage of the AC bus [3]. Compared with VSC-HVDC, the VSC-Multiple Terminal Direct Current (VSC-MTDC) could facilitate the integration of fluctuant wind power and improve the transfer efficiency and operational security [4]. Hence, with the development of power electronics technology, more WFs will be integrated into the AC grids by using VSC-MTDC.

The growing share of electricity production from WFs increases the stochastic nature of the future hybrid AC/VSC-MTDC grids [5,6]. The output power of WFs has strong uncertainties and it is difficult to accurately predict, which brings huge challenges to the secure operation of hybrid AC/VSC-MTDC grids $[7,8]$. Meanwhile, the load as the most conspicuous uncertain variable fluctuates as a function of time, weather conditions, and electricity price. With increasing price-based loads and electric vehicle charging loads, the randomness of load further increases $[9,10]$. However, the deterministic load flow 
(DLF) calculation cannot recognize these uncertainties. To properly deal with fluctuant wind power and loads, the probabilistic load flow (PLF) of AC/VSC-MTDC hybrid grids is necessary.

In general, the PLF methods can be classified into three groups: Monte Carlo Simulation (MCS) methods, analytical methods, and approximate methods [11]. MCS methods can provide the accurate results of the PLF analysis. Nevertheless, MCS methods require a large number of DLF simulations of AC/VSC-MTDC hybrid grids to reach convergence, which is extremely time-consuming. Analytical methods such as the cumulant method (CM) can improve the computational efficiency [12] but lack the ability to consider correlations among input stochastic variables (such as wind speeds and loads) [13]. By combining CM and Cholesky decomposition, a novel analytical PLF method that considers the correlation between input variables was proposed in reference [14]. However, the required linearization process of the analytical method seriously impairs its accuracy, especially due to the evident non-linear characteristics of the power flow calculation model of complex AC/VSC-MTDC grids.

In practice, approximate methods could achieve a good balance between computational accuracy and speed in PLF analysis. The point estimation method (PEM) is an excellent representative of these methods. An extended PEM was proposed to consider correlated wind speeds following diverse distributions in the probabilistic optional power flow analysis [15]. Unfortunately, it is difficult to obtain the probability distribution functions (PDFs) and cumulative distribution functions (CDFs) of the PLF results using the PEM. To estimate the PDFs or CDFs of the PLF responses, series expansion methods (SEMs) like the Gram-Charlier series and the Cornish-Fisher series can be applied in combination with the PEM output. However, SEMs may become less reliable when the probability is near 0 or 1 [16]. Furthermore, the PEM cannot accurately obtain the high-order moments of the PLF responses, leading to a decrease in the estimation accuracy of the cumulative distribution curves [17].

In reference [18], Isukapalli first proposed the stochastic response surface method (SRSM). In fact, the SRSM not only has high accuracy and computational efficiency, but it could also accurately estimate the PDFs and CDFs of the PLF responses without using SEMs. The key idea of the SRSM is to approximate the inputs and outputs of hybrid AC/DC grid power flow models considering stochastic variables, such as wind speeds and loads, by way of polynomial chaos expansion. Then, the power flow responses (such as the PDFs and CDFs of the PLF results) can be accurately calculated by the polynomial chaos expansion instead of the original DLF model, leading to an improvement in the calculation speed. However, the correlations among wind speeds have a huge impact on hybrid AC/VSC-MTDC grids, and unfortunately, cannot be handled by the traditional SRSM.

SRSM was combined with a correlation matrix method (CMM) to deal with the correlated uncertainty sources like wind speeds in the PLF calculation for the pure AC grid [19]. This is a meaningful work. Nevertheless, the CMM functions based on the assumption of a normal distribution. That is to say, using a CMM means that the input random variables (including wind speeds and diverse loads) in hybrid AC/DC grids are assumed to follow normal distributions. As is well known, depending on the locations and weather conditions, wind speeds may follow diverse distributions such as the Weibull, Burr, or Lognormal distributions [15]. Meanwhile, the behaviors of loads in the power system will be affected by resident behaviors and may not follow a normal distribution [20]. Hence, establishing the relationship between the correlated Gaussian distributions and the correlated various distributions is a good way to further improve the SRSM.

An SRSM-based PLF method for hybrid AC/VSC-MTDC grids considering correlated wind speeds and loads following diverse distributions was proposed in this paper. Compared with other methods, the main advantages of the proposed method are as follows:

(1) Random variables following diverse distributions even with correlations in the AC/VSC-MTDC hybrid grids can be properly and efficiently considered;

(2) The proposed method could keep a good balance between computational accuracy and speed in the PLF analysis of AC/VSC-MTDC grids;

(3) The means, standard deviations (STDs), PDFs, and CDFs of the PLF responses can be accurately obtained using the proposed method, without using any SEMs. 
The remainder of the paper is organized as follows. The model of a VSC is given in Section 2. Section 3 introduces the formulation and procedure of the PLF method based on the SRSM. The proposed PLF method considering stochastic variables following diverse distributions is introduced in Section 4. Section 5 presents the case studies, followed by conclusions in Section 6 .

\section{Power Flow Calculation for the AC/VSC-MTDC Hybrid Grid}

\subsection{VSC Model}

As shown in Figure 1, the $i$ th VSC in the VSC-MTDC can be expressed as a controllable voltage source $\overline{U_{c i}}=U_{c i} \angle \delta_{c i}$ with complex impedance $\overline{Z_{c i}}=R_{c i}+j X_{c i}$. Then, a transformer and a filter are connected with the converter. The filter represented by its susceptance $B_{f i}$ is connected to AC bus $i$ through the transformer represented by $\overline{Z_{t f i}}=R_{t f i}+j X_{t f i}$. Actually, the apparent power flowing from the converter and injecting to AC bus $\overline{U_{s i}}=U_{s i} \angle \delta_{s i}$, respectively, are $\overline{S_{c i}}=P_{c i}+j Q_{c i}$ and $\overline{S_{s i}}=P_{s i}+j Q_{s i}$. The equations for the power flow to AC bus can be written as in reference [21]:

$$
\left\{\begin{array}{l}
P_{s i}=-U_{s i}^{2} G_{t f i}+U_{s i} U_{f i}\left[G_{t f i} \cos \left(\delta_{s i}-\delta_{f i}\right)+B_{t f i} \sin \left(\delta_{s i}-\delta_{f i}\right)\right] \\
Q_{s i}=U_{s i}^{2} B_{t f i}+U_{s i} U_{f i}\left[G_{t f i} \sin \left(\delta_{s i}-\delta_{f i}\right)-B_{t f i} \cos \left(\delta_{s i}-\delta_{f i}\right)\right]
\end{array}\right.
$$

where $\overline{U_{f i}}=U_{f i} \angle \delta_{f i}$ is the voltage at the filter.

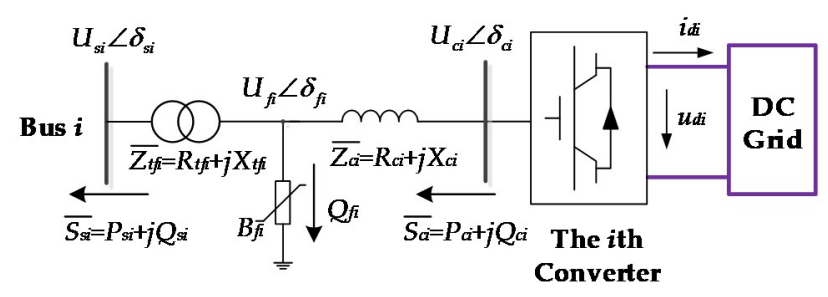

Figure 1. Steady-state model of a Voltage Source Converter (VSC) station.

Meanwhile, the equations for power flow from the $i$ th converter can be expressed as:

$$
\left\{\begin{array}{l}
P_{c i}=U_{c i}^{2} G_{c i}-U_{c i} U_{f i}\left[G_{c i} \cos \left(\delta_{f i}-\delta_{c i}\right)-B_{c i} \sin \left(\delta_{f i}-\delta_{c i}\right)\right] \\
Q_{c i}=-U_{c i}^{2} B_{c i}+U_{c i} U_{f i}\left[G_{c i} \sin \left(\delta_{f i}-\delta_{c i}\right)-B_{c i} \cos \left(\delta_{f i}-\delta_{c i}\right)\right]
\end{array}\right.
$$

The reactive power from the filter is

$$
Q_{f i}=-U_{f i}^{2} B_{f i}
$$

Generally, the real power balance of the $i$ th converter can be formulated as

$$
P_{c i}+P_{D C i}+P_{\text {Loss } \_i}=0
$$

The $P_{D C i}$ is calculated as

$$
P_{D C i}=u_{d i} \sum_{\substack{j=1 \\ j \neq i}}^{n} Y_{d i j} \times\left(u_{d i}-u_{d j}\right)
$$

where $u_{d i}$ is the DC voltage at the $i$ th converter, $Y_{d i j}$ is the conductance matrix of the DC grids.

The is $P_{\text {Loss } \_i}$ obtained by

$$
P_{\text {Loss } \_i}=K_{A}+K_{B} I_{c i}-K_{C} I_{c i}^{2}
$$


where $K_{A}, K_{B}$, and $K_{C}$ are constant parameters that are presented in reference [15], $I_{c i}$ is the AC current magnitude.

\subsection{Control Modes of VSC}

To maintain the balance of real power in the DC grids, at least one VSC, which can be viewed as an active power regulator in the VSC-MTDC, must be set to constant $u_{d i}$ control. The control modes of VSCs are presented in Table 1.

In practice, wind farms are usually connected to AC grids using the VSC-MTDC. The output power of WFs constantly fluctuates. Thus, the control modes of VSCs on the WF side (WSVSC) cannot be set to the constant control modes given in Table 1. Reference [22] presents a dual-mode control strategy (DMC) for WSVSC. There are two advantages of using a DMC for the WSVSC: the WSVSC can actively adapt the varying output of a WF, maintaining the frequency stability of the WF, and the voltage of the WSVSC AC bus is controlled to be constant, improving the voltage stability of the WF. Therefore, the DMC control mode will be applied in the WSVSC in this paper.

Table 1. Control Modes of VSCs.

\begin{tabular}{cccc}
\hline$u_{d i}-Q_{s i}$ & $u_{d i}-U_{s i}$ & $\boldsymbol{P}_{s i}-Q_{s i}$ & $\boldsymbol{P}_{s i}-\boldsymbol{U}_{s i}$ \\
\hline constant $u_{d i}, Q_{s i}$ control & constant $u_{d i}, U_{s i}$ control & constant $P_{s i}, Q_{s i}$ control & constant $P_{s i}, U_{s i}$ control \\
\hline
\end{tabular}

\subsection{Power Flow Calculation for AC/VSC-MTDC Hybrid Grids}

The popular sequential method is widely applied to solve the DLF problem of AC/VSC-MTDC hybrid grids. The specified procedures of the DLF calculation for AC/VSC-MTDC hybrid grids were introduced in Reference [21]. In fact, the DLF calculation for AC/DC hybrid grids can be expressed as a complex function with input and output variables shown as follows:

$$
\mathrm{Y}=H(\mathrm{X})
$$

where $X$ denotes input variables, including parameters of power grids, loads, and injected power from traditional generation methods and wind farms, whilst $Y$ represents output variables, including the voltages of $\mathrm{AC}$ and $\mathrm{DC}$ buses, branch power flow, and so on. If the volatility and randomness of wind power is considered, the model given above will become a PLF problem.

\section{Probabilistic Power Flow with the Stochastic Response Surface Method}

The SRSM is an effective method for calculation of the PLF of AC/VSC-MTDC hybrid grids because it promotes computational efficiency and accuracy [19]. The key idea of the SRSM lies in the application of polynomial chaos expansion with input variables following standard Gaussian distributions to simulate the response of a system like the PLF calculation of hybrid AC/VSC-MTDC grids. Hence, the SRSM method mainly includes three steps [23]: (1) Representation of input random variables; (2) functional representation of the outputs; and (3) estimation of parameters in a polynomial chaos expansion.

\subsection{Representation of Input Random Variables in Hybrid AC/VSC-MTDC Grids}

In AC/VSC-MTDC hybrid grids, wind speeds may follow different distributions such as Weibull, Burr, or Lognormal distributions; meanwhile, the loads can be affected by diverse factors and follow various distributions [24]. However, the input random variables for the SRSM must be assumed to follow standard Gaussian distributions. Thus, it is necessary to transform the random variables following diverse distributions to follow standard normal distributions by

$$
x_{i}=F_{i}^{-1}\left[\Phi\left(z_{i}\right)\right], i=1,2,3, \cdots, m
$$


where $x_{i}$ is the $i$ th element of $\mathrm{X}, F_{i}$ is the cumulative distribution function (CDF) of $x_{i}, F_{i}^{-1}$ is the inverse function of $F_{i}, z_{i}$ represents the standard normal variable, $\Phi\left(z_{i}\right)$ denotes the normal single-variable $\mathrm{CDF}$, and $m$ is the number of input random variables in the $\mathrm{AC} / \mathrm{DC}$ hybrid grid.

In practice, the method introduced above can only handle independent random variables. However, in AC/VSC-MTDC hybrid grids, the correlations among wind speeds may have a huge impact on the power system's operation. Meanwhile, the correlations between the wind speeds and loads cannot be ignored. Reference [19] introduced a CMM combined with the SRSM to deal with the correlated stochastic variables in the PLF analysis for pure AC grids. However, the CMM assumes that all input stochastic variables follow normal distributions. Wind speeds in AC/VSC-MTDC hybrid grids are affected by various complex factors, such as environmental and climatic factors, and may follow Weibull, Burr, or Lognormal distributions. Therefore, as discussed in Section 4, a new method was introduced to be used in combination with the SRSM to handle correlated input random variables following diverse distributions.

\subsection{Functional Representation of the Power Flow Model}

The Hermite polynomials in the SRSM are generally applied for functional representation. Assume that $Y=\left[y_{1}, y_{2}, \ldots, y_{d}\right]$ is the vector of system responses, like the voltages of AC and DC buses, and let $\mathrm{X}=\left[x_{1}, x_{2}, \ldots, x_{m}\right]$ denote the input random variables, such as wind speeds and loads. Meanwhile, the input stochastic vector can be represented by the independent standard normal vector $Z$. Therefore, the output of the DLF calculation for the AC/VSC-MTDC grids can be approximated by

$$
Y=a_{0}+\sum_{j_{1}=1}^{m} a_{j_{1}} H_{1}\left(z_{j_{1}}\right)+\sum_{j_{1}=1}^{m} \sum_{j_{2}=1}^{j_{1}} a_{j_{1}, j_{2}} H_{2}\left(z_{j_{1}}, z_{j_{2}}\right)+\sum_{j_{1}=1}^{m} \sum_{j_{2}=1}^{j_{1}} \sum_{j_{3}=1}^{j_{2}} a_{j_{1}, j_{2}, j_{3}} H_{3}\left(z_{j_{1}}, z_{j_{2}}, z_{j_{3}}\right)+\cdots
$$

where $z_{j_{1}}, z_{j_{2}}$, and $z_{j_{3}}$ are independent standard normal variables; $a_{j_{1}}, a_{j_{1}, j_{2}}$, and $a_{j_{1}, j_{2}, j_{3}}$ are the parameters that should be estimated; and $H_{k}$ represents a multidimensional Hermite polynomial of the order $k(k=1,2,3, \ldots)$. The actual formula of $H_{k}$ with $m$-dimension variables can be expressed by

$$
H_{k}\left(z_{j_{1}}, z_{j_{2}}, \cdots, z_{j_{m}}\right)=(-1)^{m} e^{\frac{1}{2} Z^{T} Z} \frac{\partial^{n}}{\partial_{j_{1}} \partial_{j_{2}} \cdots \partial_{j_{n}}} e^{-\frac{1}{2} Z^{T} Z}
$$

Reference [13] points out that the second-order polynomial chaos expansion has the ability to accurately handle PLF problems with high computational efficiency. Thus, the second-order polynomial chaos expansion was adopted in this paper for the PLF calculation of AC/VSC-MTDC hybrid grids, which is given by

$$
Y=a_{0,2}+\sum_{j=1}^{m} a_{j, 2} z_{j}+\sum_{j=1}^{m} a_{j j, 2}\left(u_{j}^{2}-1\right)+\sum_{j=1}^{m-1} \sum_{j>k}^{m} a_{j k, 2} z_{j} z_{k}
$$

where $a_{0,2}, a_{j, 2}, a_{j j, 2}$, and $a_{j k, 2}$ are undetermined parameters; $z_{j}$ and $z_{k}$ are standard normal variables.

\subsection{Estimation of Parameters in Polynomial Chaos Expansion}

The undetermined parameters in a polynomial chaos expansion can be estimated using the probabilistic collocation method. The key issue for the probabilistic collocation method is the selection of reasonable collocation points from the input standard normal distributions. Using Gaussian quadrature, the locations of collocation points can be selected from the roots of next-higher-order Hermite polynomials for the input random variables. For the second-order polynomial chaos expansion used in this paper, the three roots of the third-order Hermite polynomial were $0, \sqrt{3}$, and $-\sqrt{3}$.

Generally, there are three criteria for selecting the collocation points. Firstly, the collocation points should be selected to capture high-probability regions. Secondly, the collocation points should be 
selected more symmetrically on standard Gaussian distributions. Thirdly, the number of collocation points should be twice the number of the input uncertainty variables. The detailed method for the selection of collocation points is given in reference [19].

During PLF analysis for AC/VSC-MTDC hybrid grids, when the collocation points are determined, these selected collocation points must be transformed to the original space. Then, the sample points of the original space are applied to implement the DLF calculation for the AC/VSC-MTDC hybrid grids. Finally, using the least squares method, the undetermined parameters in the polynomial chaos expansion are estimated from the values of the collocation points and the results of the DLF calculation.

Once the unknown parameters are obtained, the results of the PLF calculation for the AC/DC grids, such as the PDFs of voltages and moments of branch flows, can be directly evaluated for specified Hermite polynomials instead of the original DLF model for the AC/VSC-MTDC grids. Actually, MCS or the Latin hypercube sampling method can be applied for the above purpose. It is valuable to note that this PLF calculation for AC/VSC-MTDC hybrid grids does not involve a large number of simulations using the DLF model of AC/DC grids, but only the evaluation of sample algebraic expressions, leading to an improvement in the computational efficiency.

\section{Probabilistic Power Flow for Correlated Input Variables Following Diverse Distributions}

As mentioned in Section 3.1, using the SRSM with CMM [19] requires the assumption that the correlated input stochastic variables follow normal distributions. However, in practice, for AC/VSC-MTDC hybrid grids, the wind speeds generally do not follow normal distributions and may follow Weibull, Burr, or Lognormal distributions. Meanwhile, the loads are affected by residents' behaviors and may not follow normal distributions. To remove the limitations of reference [13], the NATAF transformation was adopted in this paper.

\subsection{NATAF Transformation for Correlated Input Variables Following Different Distributions}

The wind speeds and loads in the AC/VSC-MTDC hybrid grids can be represented as a vector $\mathrm{X}=\left[x_{1}, x_{2}, \ldots, x_{m}\right]$. The CDF $F_{i}$ of $x_{i}$ and the linear correlation matrix $C_{X}$ for input variables can be estimated from historical records. The input stochastic variables following diverse distributions can be transformed into a standardized normal random vector $R=\left[r_{1}, r_{2}, \ldots, r_{m}\right]$ with the linear correlation matrix $C_{R}$ :

$$
\left\{\begin{array}{l}
\Phi\left(r_{i}\right)=F_{i}\left(x_{i}\right) \\
r_{i}=\Phi^{-1}\left[F_{i}\left(x_{i}\right)\right]
\end{array}, i=1,2,3, \cdots, m\right.
$$

where $\Phi^{-1}(-)$ denotes the inverse standard normal CDF.

Note that the linear correlation coefficient is not invariant under strictly increasing nonlinear transformations, such as the transformation of the marginal distribution. That is to say, the linear correlation matrix $C_{X}$ will change when the random variables following different distributions are transformed into standard normal distributions as described in reference [15]. Hence, it is necessary to accurately calculate $C_{R}$ using the NATAF transformation.

For $i \neq j, \rho_{r}(i, j)$ is the element of the $i$ th row and the $j$ th column of $C_{R}$. Similarly, $\rho_{x}(i, j)$ is the element of the $i$ th row and the $j$ th column of $C_{X}$. The two standard normal variables $r_{i}$ and $r_{j}$ correspond to two input random variables $x_{i}$ and $x_{j}$ of the AC/DC hybrid grids. Based on the definition of mathematical expectation, $E\left[x_{i} x_{j}\right]$ can be expressed as

$$
E\left[x_{i} x_{j}\right]=E\left[F_{i}^{-1}\left[\Phi\left(r_{i}\right)\right], F_{j}^{-1}\left[\Phi\left(r_{j}\right)\right]\right]=\int_{-\infty}^{+\infty} \int_{-\infty}^{+\infty} F_{i}^{-1}\left[\Phi\left(r_{i}\right)\right], F_{j}^{-1}\left[\Phi\left(r_{j}\right)\right] \times \varphi_{\rho_{r}(i, j)}\left(r_{i}, r_{j}\right) d r_{i} d r_{j}
$$

where $\varphi_{\rho_{r}(i, j)}$ is the standard joint normal PDF with the correlation coefficient $\rho_{r}(i, j)$. Let $\rho=\rho_{r}(i, j)$; the joint PDF $\varphi_{\rho_{r}(i, j)}$ can be expressed as 


$$
\varphi_{\rho_{r}(i, j)}\left(r_{i}, r_{j}\right)=\frac{e^{-\left(r_{i}^{2}-2 \rho r_{i} r_{j}+r_{i}^{2}\right) / 2\left(1-\rho^{2}\right)}}{2 \pi \sqrt{1-\rho^{2}}}
$$

Based on Equations (12)-(14), the linear correlation coefficient $\rho_{x}(i, j)$ between the input variables can be represented by $\rho_{r}(i, j)$, which is given by

$$
\rho_{x}(i, j)=\frac{\int_{-\infty}^{+\infty} \int_{-\infty}^{+\infty} F_{i}^{-1}\left[\Phi\left(r_{i}\right)\right], F_{j}^{-1}\left[\Phi\left(r_{j}\right)\right] \times \varphi_{\rho_{r}(i, j)}\left(r_{i}, r_{j}\right) d r_{i} d r_{j}-\mu_{i} \mu_{j}}{\sigma_{i} \sigma_{j}}
$$

where $\mu_{i}, \mu_{j}, \sigma_{i}$, and $\sigma_{j}$ are the means and standard deviations of $x_{i}$ and $x_{j}$. As shown in Equation (15), the relationship function between $\rho_{x}(i, j)$ and $\rho_{r}(i, j)$ is established. Although it is a complex nonlinear function, Reference [17] presents an efficient search method for obtaining $\rho_{r}(i, j)$ based on $\rho_{x}(i, j)$.

After the correlation matrix $C_{R}$ is obtained, it can be factorized by using the Cholesky decomposition as follows:

$$
C_{R}=L L^{T}
$$

where $L$ represents a lower triangular matrix. Hence, the correlated standard normal vector $R$ can be obtained by

$$
R=L^{-1} Z
$$

Then, the correlated stochastic variables following different distributions (such as wind speeds and loads) can be obtained using Equation (8).

\subsection{Procedure for Solving the PLF of AC/DC Hybrid Grids Considering Correlated Wind Speeds and Loads}

When combined with the NATAF transformation, the SRSM can deal with correlated random variables following different distributions in the PLF calculations for the AC/VSC-MTDC hybrid grids. The procedure for solving the PLF calculation is summarized below.

(1) Input the parameters of the AC/VSC-MTDC hybrid grid, random variables (such as wind speeds and loads), and the correlation matrix $C_{X}$.

(2) Calculate the correlation matrix $C_{R}$ of $\mathrm{R}$ from $C_{X}$ based on Equation (15).

(3) Calculate the lower triangular matrix $L$ using Equation (16).

(4) Select the collocation points from the independent standard normal vector Z .

(5) Compute the correlated collocation points in the standard normal space using Equation (17).

(6) Transform the collocation points with the correlation matrix $C_{R}$ in the standard normal space to the original space, following the different distributions, using Equation (8).

(7) Feed the collocation points in the original space to the DLF calculation of the AC/VSC-MTDC hybrid grid and save the results.

(8) Determine the undetermined parameters in the polynomial chaos expansion.

(9) Represent the power flow responses $Y$ using polynomial chaos expansion and estimate the PDFs, $\mathrm{CDFs}$, and the moments of $\mathrm{Y}$.

\section{Case Study}

\subsection{System, Data, and Study Scenarios}

The performance of the proposed PLF method was tested using a modified IEEE 118-bus system with a VSC-MTDC. As shown in Figure 2, the wind farms $W_{1}, W F_{2}$, and $W_{3}$ were connected to the AC grid at bus 24 and bus 35 using a five-terminal DC grid incorporating VSCs. Meanwhile, the other three wind farms $\mathrm{WF}_{4}, \mathrm{WF}_{5}$, and $\mathrm{WF}_{6}$ were directly connected to the AC grid at bus 54, bus 115, and bus 45 , respectively. The parameters of the IEEE 118-bus system can be found in Matpower 6.0 as in reference [25]. The parameters of the VSCs and DC lines are given in reference [3]. In this paper, 
the control modes of the VSCs are presented in Table 2. Note that the base capacities of the AC and DC grids were set to be 100 MVA.

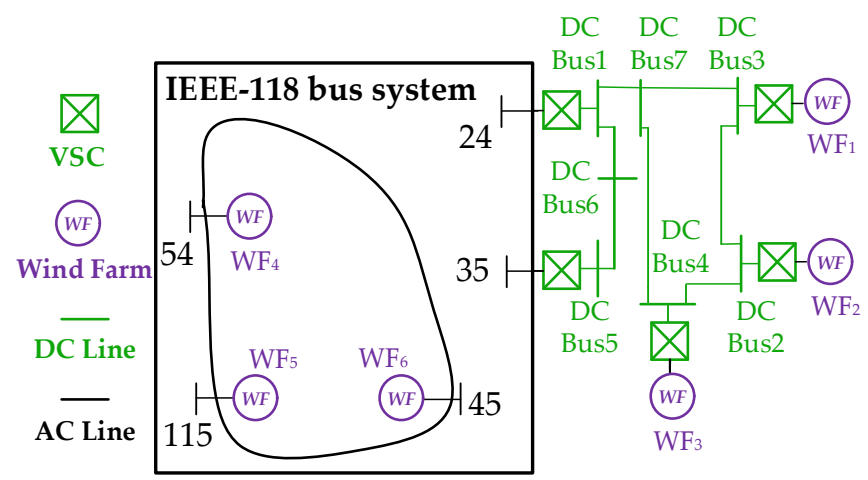

Figure 2. Modified IEEE 118-bus system with a VSC-Multiple Terminal Direct Current (VSC-MTDC) system.

Table 2. Control Modes of VSCs.

\begin{tabular}{cccccc}
\hline DC Bus & Control Modes & $\begin{array}{c}u_{d i} \\
(\mathbf{p . u})\end{array}$ & $\begin{array}{c}U_{s i} \\
(\mathbf{p . u})\end{array}$ & $\begin{array}{c}\boldsymbol{P}_{s i} \\
(\mathbf{p . u})\end{array}$ & $\begin{array}{c}Q_{s i} \\
(\mathbf{p} . \mathbf{u})\end{array}$ \\
\hline 1 & $u_{d i}-Q_{s i}$ & 1.00 & $\backslash$ & $\backslash$ & 0.40 \\
2 & $\mathrm{DMC}$ & $\backslash$ & 1.05 & $\backslash$ & $\backslash$ \\
3 & $\mathrm{DMC}$ & $\backslash$ & 1.05 & $\backslash$ & $\backslash$ \\
4 & $\mathrm{DMC}$ & $\backslash$ & 1.05 & $\backslash$ & $\backslash$ \\
5 & $P_{s i}-Q_{s i}$ & $\backslash$ & $\backslash$ & 0.98 & 0.3 \\
\hline
\end{tabular}

The WF output model presented in reference [24] was used for all WFs in this case study. The rated capacities of the WFs were set to be $80 \mathrm{MW}$, and the power factors of the WFs were set to be 0.95 . It was assumed that the cut-in, rated, and cut-out wind speeds of the WFs were $3 \mathrm{~m} / \mathrm{s}, 11 \mathrm{~m} / \mathrm{s}$, and $24 \mathrm{~m} / \mathrm{s}$, respectively. The correlation coefficients between the wind speeds of different WFs are given in Table 3 . It was assumed that the correlation coefficients among the bus loads were 0.1 and that the correlation coefficients between the wind speeds and loads were -0.1 .

Table 3. The correlation coefficients among wind speeds.

\begin{tabular}{ccccccc}
\hline Wind Farms & $\mathbf{W F}_{\mathbf{1}}$ & $\mathbf{W F}_{\mathbf{2}}$ & $\mathbf{W F}_{\mathbf{3}}$ & $\mathbf{W F}_{\mathbf{4}}$ & $\mathbf{W F}_{\mathbf{5}}$ & $\mathbf{W F}_{\mathbf{6}}$ \\
\hline $\mathrm{WF}_{1}$ & 1 & 0.6 & 0.55 & 0.12 & 0.23 & 0.34 \\
$\mathrm{WF}_{2}$ & 0.6 & 1 & 0.66 & 0.24 & 0.23 & 0.34 \\
$\mathrm{WF}_{3}$ & 0.55 & 0.66 & 1 & 0.72 & 0.65 & 0.44 \\
$\mathrm{WF}_{4}$ & 0.12 & 0.24 & 0.72 & 1 & 0.55 & 0.57 \\
$\mathrm{WF}_{5}$ & 0.23 & 0.23 & 0.65 & 0.55 & 1 & 0.3 \\
$\mathrm{WF}_{6}$ & 0.34 & 0.34 & 0.44 & 0.57 & 0.3 & 1 \\
\hline
\end{tabular}

As is well known, wind speeds are affected by various factors and may follow Weibull, Burr, or Lognormal distributions. Meanwhile, some resident loads in a practical power system may follow a Weibull distribution or Gaussian distribution [20]. To demonstrate the effectiveness of the proposed PLF method, four operation scenarios of the hybrid AC/VSC-MTDC grids were defined, as shown in Table 4. All the uncertain sources followed normal distributions in operation scenario 1, while the wind speeds at the wind farms $\mathrm{WF}_{1}, \mathrm{WF}_{2}$, and $\mathrm{WF}_{3}$ in operation scenario 2 were assumed to follow a Weibull distribution. In operation scenario 3 , wind speeds at the different wind farms followed various distributions. Furthermore, in operation scenario 4, different types of loads were considered, and loads at buses $11,12,13,14,15,39,40,41,42,43,44,74,75,76,77,78,110$, and 115 were assumed to follow a 
Weibull distribution. Note that the historical records of wind speeds in the four different operation scenarios can be accessed in reference [26].

Table 4. The operation scenarios of hybrid AC/VSC-MTDC grids.

\begin{tabular}{ccccc}
\hline Uncertainty Sources & $\begin{array}{c}\text { Operation } \\
\text { Scenario 1 }\end{array}$ & $\begin{array}{c}\text { Operation } \\
\text { Scenario 2 }\end{array}$ & $\begin{array}{c}\text { Operation } \\
\text { Scenario 3 }\end{array}$ & $\begin{array}{c}\text { Operation } \\
\text { Scenario 4 }\end{array}$ \\
\hline WF1 & Normal & Weibull & Weibull & Weibull \\
\hline WF2 & Normal & Weibull & Lognormal & Lognormal \\
\hline WF3 & Normal & Weibull & Burr & Burr \\
\hline WF4 & Normal & Normal & Weibull & Weibull \\
\hline WF5 & Normal & Normal & Lognormal & Lognormal \\
\hline WF6 & Normal & Normal & Burr & Burr \\
\hline $\begin{array}{c}\text { Loads at buses } 11,12,13,14,15,39,40,41, \\
42,43,44,74,75,76,77,78,110, \text { and } 115\end{array}$ & Normal & Normal & Normal & \multirow{2}{*}{ Weibull } \\
\hline Other loads & Normal & Normal & Normal & Normal \\
\hline
\end{tabular}

To verify the accuracy and efficiency of the proposed PLF method, the MCS method-based NATAF transformation was used to provide the reference results (note that this method was denoted as MCS-NATAF in this paper). The MCS generated 20,000 sample points from various correlated distributions, which was sufficient to yield reliable PLF results. To further demonstrate the superiority of the proposed PLF method in handling correlated random variables following different distributions, the results were compared with those from the following methods:

- In reference [14], combining the CM and Cholesky decomposition, an improved CM based PLF method was developed to consider the correlated random variables; this was denoted as CM-CD in this paper.

- In reference [15], an extended PEM based on the NATAF transformation was introduced to deal with various random variables with correlations; this was denoted as PEM-NATAF in this paper.

- $\quad$ SRSM was combined with a CMM to deal with correlated uncertainty sources in reference [19]; this was denoted as SRSM-CMM in this paper.

Note that the SRSM and NATAF transformation were combined to form the proposed PLF method in this paper; we denoted this as SRSM-NATAF.

\subsection{Performance Evaluation}

The average errors of the mean values of the DC bus voltages in the four different operational scenarios are given in Table 5. The errors of the standard deviation (STD) values of the DC bus voltages in the four different operational scenarios are shown in Figure 3. Meanwhile, Table 6 presents the errors of the mean and STD values of the active power losses from the AC grid in the four operational scenarios. Note that the errors in the figures and tables refer to the percentage of relative error against the results obtained by the MCS-NATAF method.

As shown in Tables 5 and 6 and Figure 3, the responses of the PLF analysis for the AC/VSC-MTDC hybrid grids obtained by the CM-CD method present huge errors in all operation scenarios. In the four operation scenarios, the errors of the mean and STD values of the active power losses from the AC grid obtained by CM-CD, given respectively, were $3.68 \%$ and $14.13 \%$ (for operation scenario 1 ), $4.56 \%$ and $16.37 \%$ (for operation scenario 2 ), $4.89 \%$ and $17.34 \%$ (for operation scenario 3 ), and $4.92 \%$ and $17.89 \%$ (for operation scenario 4). The average errors of the mean and STD values of the DC bus voltages obtained by the CM-CD method, respectively, were $1.26 \%$ and $5.53 \%$ (for operational scenario 1 ), $1.41 \%$ and $6.28 \%$ (for operational scenario 2), $1.67 \%$ and $7.45 \%$ (for operational scenario 3 ), and $1.69 \%$ and $8.35 \%$ (for operational scenario 4). The improved cumulant method (CM-CD) will become less accurate if the system (such as a complex AC/VSC-MTDC hybrid grid) moves far from the linear region of 
behavior. The calculation errors of the CM-CD could further increase, if the system operates in stressed conditions, such as random variables following diverse distributions even with correlations.

Table 5. The average errors of mean values of the DC bus voltages in the four different operational scenarios examined.

\begin{tabular}{ccccc}
\hline Algorithm & $\begin{array}{c}\text { Operation } \\
\text { Scenario 1 }\end{array}$ & $\begin{array}{c}\text { Operation } \\
\text { Scenario 2 }\end{array}$ & $\begin{array}{c}\text { Operation } \\
\text { Scenario 3 }\end{array}$ & $\begin{array}{c}\text { Operation } \\
\text { Scenario 4 }\end{array}$ \\
\hline SRSM-NATAF (\%) & 0.31 & 0.33 & 0.38 & 0.42 \\
SRSM-CMM (\%) & 0.30 & 0.89 & 1.32 & 1.43 \\
CM-CD (\%) & 1.26 & 1.41 & 1.67 & 1.69 \\
PEM-NATAF (\%) & 0.32 & 0.32 & 0.36 & 0.45 \\
\hline
\end{tabular}

Table 6. The errors of the mean and standard deviation values of the active power losses from the AC grid in the four different operational scenarios examined.

\begin{tabular}{cccccc}
\hline Algorithm & $\begin{array}{c}\text { Errors } \\
\mathbf{( \% )}\end{array}$ & $\begin{array}{c}\text { Operation } \\
\text { Scenario 1 }\end{array}$ & $\begin{array}{c}\text { Operation } \\
\text { Scenario 2 }\end{array}$ & $\begin{array}{c}\text { Operation } \\
\text { Scenario 3 }\end{array}$ & $\begin{array}{c}\text { Operation } \\
\text { Scenario 4 }\end{array}$ \\
\hline \multirow{2}{*}{ SRSM-NATAF } & Mean & 1.23 & 1.89 & 2.55 & 2.89 \\
& STD & 3.22 & 3.45 & 4.12 & 4.59 \\
\hline \multirow{2}{*}{ SRSM-CMM } & Mean & 1.26 & 4.23 & 5.88 & 6.89 \\
& STD & 3.29 & 6.24 & 10.19 & 16.56 \\
\hline \multirow{2}{*}{ CM-CD } & Mean & 3.68 & 4.56 & 4.89 & 4.92 \\
& STD & 14.13 & 16.37 & 17.34 & 17.89 \\
\hline \multirow{2}{*}{ PEM-NATAF } & Mean & 1.28 & 1.96 & 2.83 & 2.95 \\
& STD & 3.18 & 3.66 & 4.39 & 4.75 \\
\hline
\end{tabular}

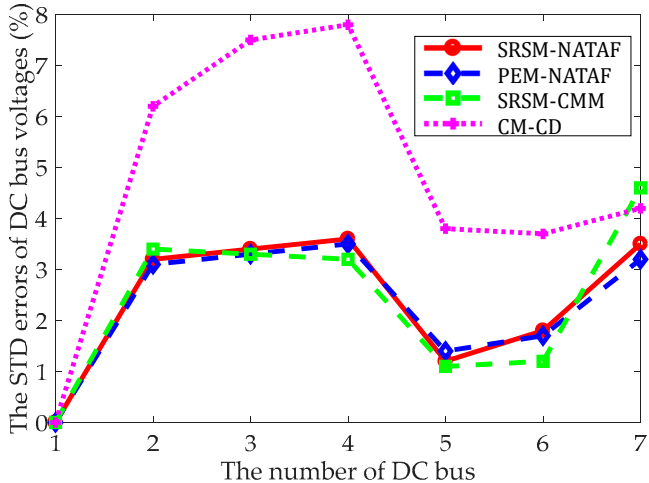

(A) Operation scenario 1

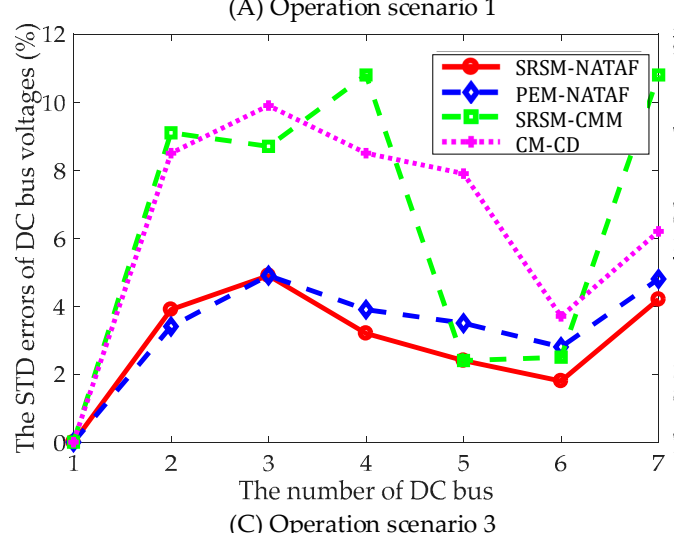

(C) Operation scenario 3

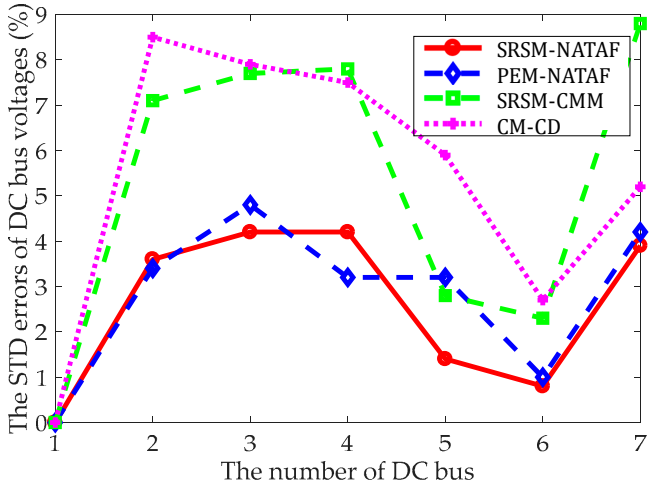

(B) Operation scenario 2

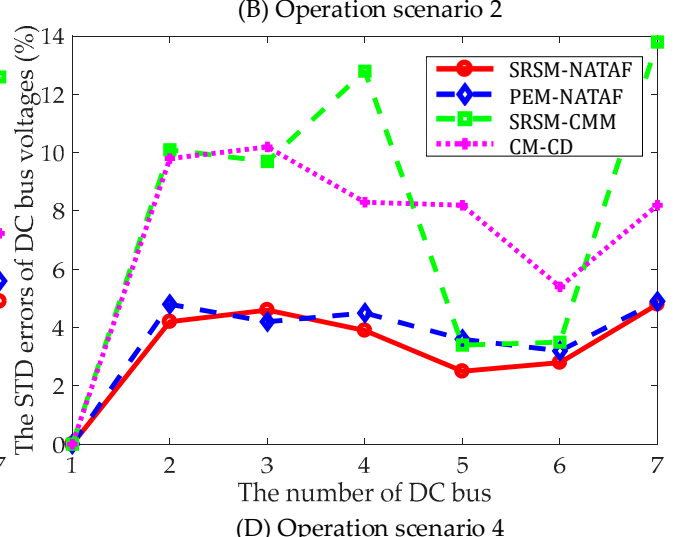

Figure 3. The standard deviation (STD) errors of the DC bus voltages in the four different operational scenarios. 
In operation scenario 1, both the mean and STD error values obtained using SRSM-NATAF, SRSM-CMM, and PEM-NATAF were almost the same. The average errors of the mean and STD values of the DC bus voltages obtained by SRSM-NATAF, SRSM-CMM, and PEM-NATAF were $0.31 \%, 0.30 \%$, and $0.32 \%$ (for the means), and $2.78 \%, 2.8 \%$, and $2.73 \%$ (for the STDs). The errors of the mean and STD values of the active power losses from the AC grid, given respectively, were $1.23 \%$ and $3.22 \%$ (for SRSM-NATAF), $1.26 \%$ and 3.29\% (for SRSM-CMM), and 1.28\% and 3.18\% (for PEM-NATAF). The reason for this was that all the input random variables in operational scenario 1 were assumed to follow normal distributions, and the SRSM-NATAF, SRSM-CMM, and PEM-NATAF methods all had a good ability to deal with correlated random variables following normal distributions in a hybrid AC/VSC-MTDC grid.

Nevertheless, wind speeds in a practical power system may not follow normal distributions but may follow diverse distributions, even with correlations. In operation scenarios 2 and 3 (wherein the wind speeds were assumed to follow various distributions), it could be observed from Tables 5 and 6 , and Figure 3, that the relative errors obtained using SRSM-NATAF and PEM-NATAF were much smaller than those obtained using SRSM-CMM. For example, in operation scenario 2, the STD errors in the DC bus voltage obtained using SRSM-NATAF, PEM-NATAF, and SRSM-CMM were 3.02\%, $3.3 \%$, and $6.08 \%$, respectively. Meanwhile, in operation scenario 3, the STD errors in the DC bus voltage were $3.41 \%, 3.88 \%$, and $7.38 \%$. SRSM-CMM cannot accurately consider different correlated distributions, resulting in a decrease in the computational accuracy of the PLF calculation. However, the proposed SRSM-NATAF method could solve this problem. It is of value to note that the calculation errors obtained using SRSM-NATAF were smaller than those obtained using PEM-NATAF.

In operation scenario 4, different types of loads were considered. In AC/VSC-MTDC hybrid grids, the number of correlated random variables following diverse distributions further increases compared with that in operation scenario 3. Hence, the computational errors from the SRSM-CMM method increase as well. In operation scenarios 3 and 4, the SRSM-CMM method's errors in the mean and STD values of the active power losses from the AC grid, given respectively, were $5.88 \%$ and $10.19 \%$ (for operation scenario 3 ), and $6.89 \%$ and $16.56 \%$ (for operation scenario 4 ). However, the proposed SRSM-NATAF method can accurately handle different distributions with correlations in the PLF analysis. For example, in operation scenario 4, the average errors in the mean and STD values of the DC bus voltage obtained by SRSM-NATAF were $0.42 \%$ and $3.8 \%$, respectively, and the errors in the mean and STD values of the active power losses from the AC grid obtained using SRSM-NATAF were $2.89 \%$ and $4.59 \%$, respectively. The key reason for this lay in the fact that CMM, based on the assumption of normal distribution, will result in inaccurate conclusions in the PLF analysis. However, the results in Tables 5 and 6, and Figure 3, show that the proposed SRSM-NATAF method was quite effective in all examined operation scenarios.

The cumulative probability curves of the voltage magnitude at bus 24 in the four different operation scenarios are shown in Figure 4. Although the cumulants and moments of the PLF output could be respectively obtained using PEM-NATAF and CM-CD, these two methods had no ability to directly estimate the PDFs and CDFs of the PLF results. Therefore, PEM-NATAF and CM-CD were combined with the popular Gram-Charlier series expansion to estimate the CDFs of the PLF responses.

It can be seen from Figure 4 that the results obtained using SRSM-CMM, CM-CD, and PEM-NATAF were obviously biased compared with those obtained by using MCS-NATAF. The reasons for this were as follows: (1) SRSM-CMM could not accurately consider the correlated different distributions, leading to inaccurate results of the PLF analysis; (2) PEM-NATAF could not accurately estimate the high-order moments of the PLF responses, resulting in poor accuracy of the cumulative probability curves; and (3) CM-CD assumed that the original DLF model was linear, which would impair its accuracy. Furthermore, as shown in Figure 4, the cumulative probability curves obtained by CM-CD and PEM-NATAF in operation scenarios 3 and 4 were evidently biased when the probability was near 0 or 1 . The key reason lay in that SEMs, like the Gram-Charlier series, may become unreliable when the probability was near 0 or 1 [16]. Compared with those from SRSM-CMM, CM-CD, 
and PEM-NATAF, the cumulative probability curves obtained using the proposed SRSM-NATAF method were much closer to those obtained using the MCS-NATAF. Hence, by using the proposed SRSM-NATAF method, the means, STDs, and the cumulative probability curves of the PLF results could be accurately obtained.

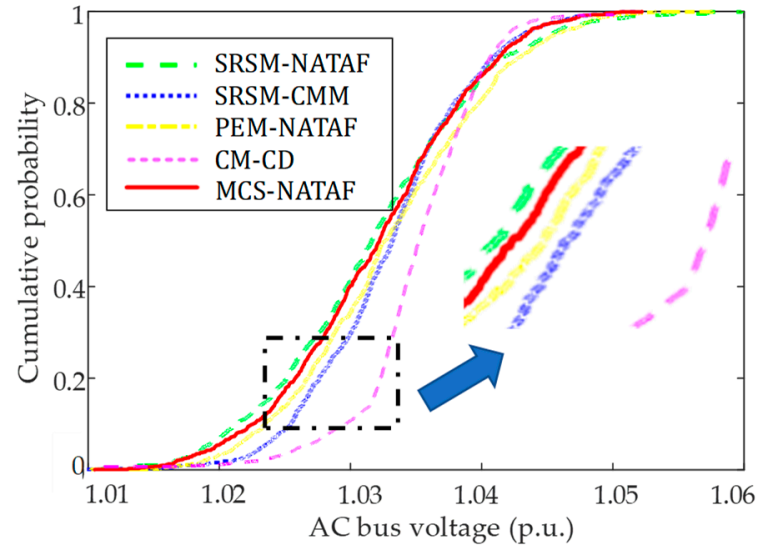

(A) Operation scenario 1

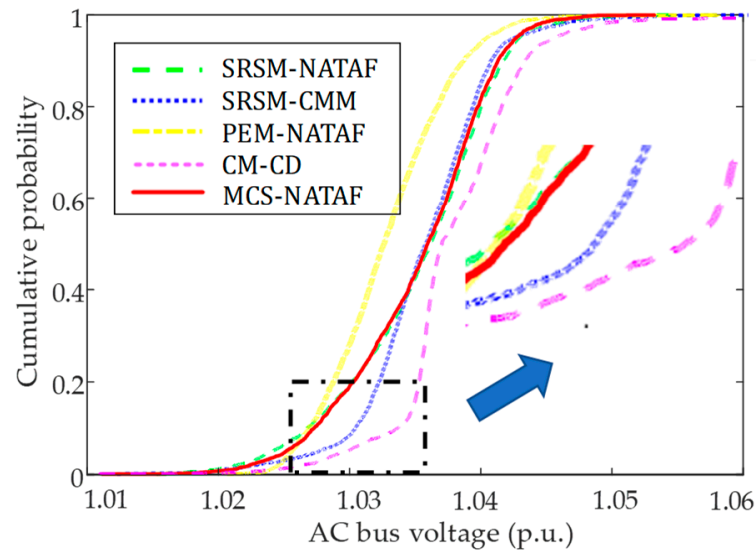

(C) Operation scenario 3

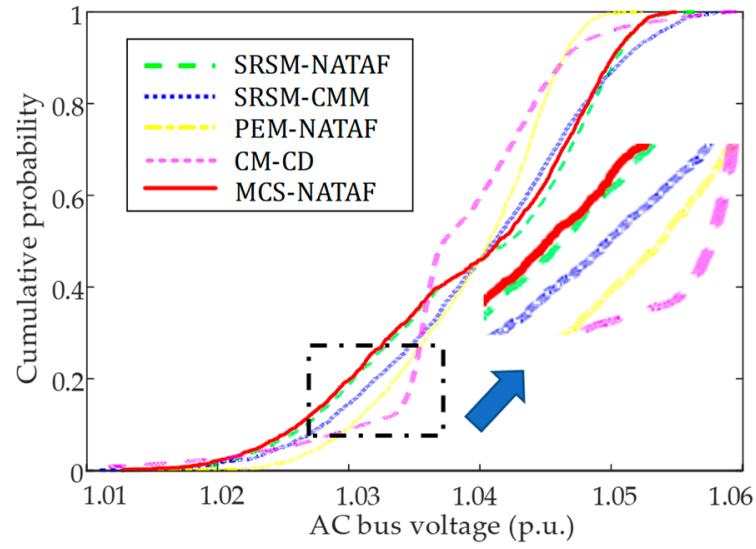

(B) Operation scenario 2

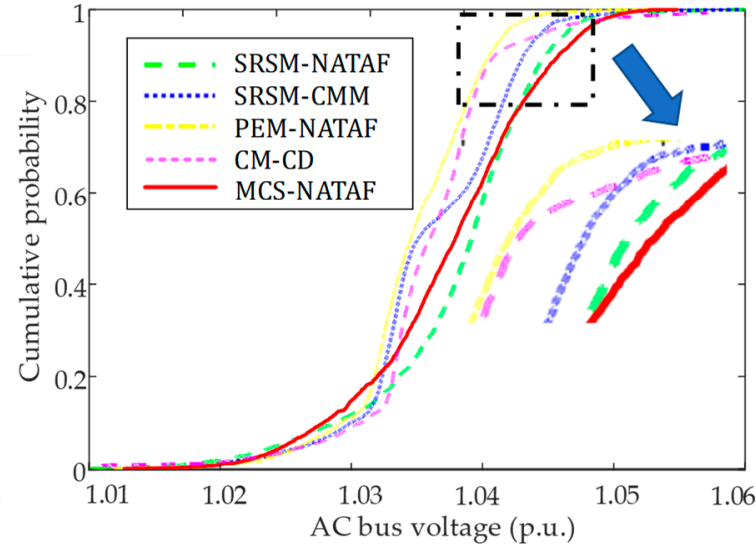

(D) Operation scenario 4

Figure 4. The cumulative probability curves of the voltage magnitude at bus 28 in the four different operation scenarios.

\subsection{Computational Efficiency}

The CPU usage times for the PLF analysis of the modified IEEE 118-bus system under the four different operation scenarios using the MCS-NATAF, SRSM-CMM, PEM-NATAF, CM-CD, and the proposed SRSM-NATAF methods are shown in Table 7. The average CPU usage times for the MCS-NATAF, CM-CD, PEM-NATAF, SRSM-CMM, and the proposed SRSM-NATAF method were $3757.89 \mathrm{~s}, 24.80 \mathrm{~s}, 33.81 \mathrm{~s}, 33.65 \mathrm{~s}$, and $33.82 \mathrm{~s}$, respectively. Although CM-CD could accelerate the PLF analysis, the required linearization process of this method seriously impaired its accuracy, especially for the complex AC/VSC-MTDC hybrid grids.

The CPU usage times of the PEM-NATAF, SRSM-CMM, and the proposed SRSM-NATAF method were almost the same. Compared with the MSC-NATAF, the proposed SRSM-NATAF method allowed an extreme reduction in the calculation time required for the PLF analysis of AC/DC hybrid grids. Hence, the proposed SRSM-NATAF method not only had good computational accuracy but it also had high computational efficiency. Note that all operation scenarios were implemented in MATLAB on an Intel i5 $3.30 \mathrm{GHz}$ PC with $8 \mathrm{~GB}$ RAM. Meanwhile, the AC power flow was solved by Matpower as in Reference [25]. 
Table 7. A comparison of computing time for the four different operation scenarios.

\begin{tabular}{ccccc}
\hline Algorithm & $\begin{array}{c}\text { Operation } \\
\text { Scenario 1 }\end{array}$ & $\begin{array}{c}\text { Operation } \\
\text { Scenario 2 }\end{array}$ & $\begin{array}{c}\text { Operation } \\
\text { Scenario 3 }\end{array}$ & $\begin{array}{c}\text { Operation } \\
\text { Scenario 4 }\end{array}$ \\
\hline MCS-NATAF (s) & 3741.56 & 3742.13 & 3757.99 & 3789.89 \\
SRSM-NATAF (s) & 33.48 & 33.53 & 33.95 & 34.29 \\
SRSM-CMM (s) & 33.45 & 33.48 & 33.75 & 33.93 \\
CM-CD (s) & 24.67 & 24.72 & 24.84 & 24.98 \\
PEM-NATAF (s) & 33.46 & 33.51 & 33.93 & 34.35 \\
\hline
\end{tabular}

\section{Conclusions}

A new PLF method based on the SRSM and NATAF transformation for AC/VSC-MTDC hybrid grids was proposed in this paper. The means, STDs, PDFs, and the CDFs of the PLF responses could be accurately and efficiently estimated using the proposed SRSM-NATAF method, without any series expansion methods. Furthermore, correlated random variables following diverse distributions could be accurately considered using the proposed method for the PLF analysis.

A modified IEEE 118-bus system under four different operation scenarios was applied to verify the correctness and effectiveness of the proposed method. The results indicated that diverse distributions with correlations could be properly dealt with by the proposed method, which led to PLF results with a higher accuracy than those calculated using the SRSM-CMM method, which assumed a normal distribution and the CM-CD method which assumed that the DLF model was linear. Unlike the PEM-NATAF, the proposed method could accurately estimate the PDFs and CDFs without using any series expansion methods. Actually, the orders of polynomial chaos expansions will affect accuracy and speed of the PLF analysis. Seeking more advanced SRSM techniques to further improve the performance of the PLF methods will be the future extension based on the work discussed herein.

Author Contributions: Formal analysis, Z.Z. and S.L.; Investigation, Z.Z., S.L., and S.P.; Methodology, Z.Z., S.L., and S.P.; Software, S.L.; Supervision, Z.Z.; Validation, S.P.; Writing一original draft, Z.Z. and S.L.; Writing一review \& editing, Z.Z. and S.P.

Funding: This research was funded by National Natural Science Foundation of China, grant number 51867017.

Conflicts of Interest: The authors declare no conflict of interest.

\section{References}

1. Li, R.; Bozhko, S.; Asher, G. Frequency Control Design for Offshore Wind Farm Grid with LCC-HVDC Link Connection. IEEE Trans. Power Electron. 2008, 23, 1085-1092. [CrossRef]

2. Baradar, M.; Ghandhari, M. A Multi-Option Unified Power Flow Approach for Hybrid AC/DC Grids Incorporating Multi-Terminal VSC-HVDC. IEEE Trans. Power Syst. 2013, 28, 2376-2383. [CrossRef]

3. Chai, R.; Zhang, B.; Dou, J.; Hao, J.; Zheng, T. Unified Power Flow Algorithm Based on the NR Method for Hybrid AC/DC Grids Incorporating VSCs. IEEE Trans. Power Syst. 2016, 31, 4310-4318. [CrossRef]

4. Che, Y.; Li, W.; Li, X.; Zhou, J.; Li, S.; Xi, X. An Improved Coordinated Control Strategy for PV System Integration with VSC-MVDC Technology. Energies 2017, 10, 1670. [CrossRef]

5. Zhang, X.; Guo, Z.; Chen, W. Probabilistic Power Flow Method Considering Continuous and Discrete Variables. Energies 2017, 10, 590. [CrossRef]

6. González-Aparicio, I.; Zucker, A. Impact of wind power uncertainty forecasting on the market integration of wind energy in Spain. Appl. Energy 2015, 159, 334-349. [CrossRef]

7. Jin, T.; Tian, Z. Uncertainty analysis for wind energy production with dynamic power curves. In Proceedings of the IEEE International Conference on Probabilistic Methods Applied to Power Systems, Singapore, 14-17 June 2010.

8. Hosseinzadeh, M.; Salmasi, F.R. Robust Optimal Power Management System for a Hybrid AC/DC Micro-Grid. IEEE Trans. Sustain. Energy 2015, 6, 675-687. [CrossRef] 
9. Hernández, J.C.; Ruizrodriguez, F.J.; Jurado, F. Technical impact of photovoltaic-distributed generation on radial distribution systems: Stochastic simulations for a feeder in Spain. Int. J. Electr. Power Energy Syst. 2013, 50, 25-32. [CrossRef]

10. Ruiz-Rodriguez, F.J.; Hernández Jesus, C.; Jurado, F. Voltage behaviour in radial distribution systems under the uncertainties of photovoltaic systems and electric vehicle charging loads. Int. Trans. Electr. Energy Syst. 2017. [CrossRef]

11. Ni, F.; Nguyen, P.; Cobben, J.F.G. Basis-Adaptive Sparse Polynomial Chaos Expansion for Probabilistic Power Flow. IEEE Trans. Power Syst. 2017, 32, 42-53. [CrossRef]

12. Yu, H.; Chung, C.Y.; Wong, K.P.; Lee, H.W.; Zhang, J.H. Probabilistic Load Flow Evaluation With Hybrid Latin Hypercube Sampling and Cholesky Decomposition. IEEE Trans. Power Syst. 2009, 24, 661-667. [CrossRef]

13. Allan, R.; Billinton, R. Probabilistic assessment of power systems. Proc. IEEE 2000, 88, 140-162. [CrossRef]

14. Cai, D.; Chen, J.; Shi, D.; Duan, X.; Li, H.; Yao, M. Enhancements to the Cumulant Method for probabilistic load flow studies. In Proceedings of the 2012 IEEE Power and Energy Society General Meeting, San Diego, CA, USA, 22-26 July 2012.

15. Li, Y.; Li, W.; Yan, W.; Yu, J.; Zhao, X. Probabilistic Optimal Power Flow Considering Correlations of Wind Speeds Following Different Distributions. IEEE Trans. Power Syst. 2014, 29, 1847-1854. [CrossRef]

16. Fan, M.; Vittal, V.; Heydt, G.T.; Ayyanar, R. Probabilistic Power Flow Studies for Transmission Systems With Photovoltaic Generation Using Cumulants. IEEE Trans. Power Syst. 2012, 27, 2251-2261. [CrossRef]

17. Prusty, B.R.; Jena, D. A critical review on probabilistic load flow studies in uncertainty constrained power systems with photovoltaic generation and a new approach. Renew. Sustain. Energy Rev. 2017, 69, 1286-1302. [CrossRef]

18. Isukapalli, S.S.; Roy, A.; Georgopoulos, P.G. Stochastic Response Surface Methods for Uncertainty Propagation: Application to Environmental and Biological Systems. Risk Anal. 2010, 18, 351-363. [CrossRef]

19. Ren, Z.; Li, W.; Billinton, R.; Yan, W. Probabilistic Power Flow Analysis Based on the Stochastic Response Surface Method. IEEE Trans. Power Syst. 2016, 31, 2307-2315. [CrossRef]

20. Irwin, G.W.; Monteith, W.; Beattie, W.C. Statistical electricity demand modelling from consumer billing data. IEEE Proc. Gener. Transm. Distrib. 2008, 133, 328-335. [CrossRef]

21. Beerten, J.; Cole, S.; Belmans, R. Generalized Steady-State VSC MTDC Model for Sequential AC/DC Power Flow Algorithms. IEEE Trans. Power Syst. 2012, 27, 821-829. [CrossRef]

22. Guan, L.; Fan, X.; Liu, Y.; Wu, Q.H. Dual-Mode Control of AC/VSC-HVDC Hybrid Transmission Systems with Wind Power Integrated. IEEE Trans. Power Deliv. 2015, 30, 1686-1693. [CrossRef]

23. Isukapalli, S.S. Uncertainty Analysis of Transport Transformation Models; Rutgers Univ.: New Brunswick, NJ, USA, 1999.

24. Qin, Z.; Li, W.; Xiong, X. Generation System Reliability Evaluation Incorporating Correlations of Wind Speeds With Different Distributions. IEEE Trans. Power Syst. 2013, 28, 551-558. [CrossRef]

25. Zimmerman, R.D.; Murillo-Sanchez, C.E.; Thomas, R.J. MATPOWER: Steady-State Operations, Planning, and Analysis Tools for Power Systems Research and Education. IEEE Trans. Power Syst. 2011, 26, 12-19. [CrossRef]

26. National Meteorological Information Center. Available online: http:/ / data.cma.cn/ (accessed on 6 October 2018). 